\title{
Confidence and curvature estimation of curvilinear structures in 3-D
}

\author{
P. Bakker, L.J. van Vliet, P.W. Verbeek \\ Pattern Recognition Group, \\ Department of Applied Physics, \\ Delft University of Technology, \\ Lorentzweg 1, 2628 CJ, Delft, The Netherlands, \\ \{peterb,lucas,piet\}@ph.tn.tudelft.nl
}

\begin{abstract}
In this paper we present a new method for estimating confidence and curvature of 3-D curvilinear structures. The gradient structure tensor (GST) models shift-invariance. The eigenstructure of the tensor allows estimation of local dimensionality, orientation, and the corresponding confidence value. Local rotational invariance, which occurs often in images, causes a lower confidence estimate. This underestimation can be corrected for by a parabolic deformation of the data, in such a way that it becomes translational invariant. We show that the optimal deformation can be found analytically and yields a local curvature estimate as a valuable by-product. We tested our new method on synthetic images and applied it to the detection of channels in 3-D seismic data.
\end{abstract}

\section{Introduction}

Translation invariant structures in 3-D images can have three different dimensionalities. Isotrope structures (3-D) with translation invariance in all orientations, plane-like structures (2-D) with translation invariance in a plane, and line-like structures (1-D) with translation invariance along one orientation.

A mathematical model for translation invariant structures is given by the structure tensor. The eigenvectors of the tensor yield a robust estimator for local orientation $[1,2,3]$. In addition, the eigenvalues contain information about the resemblance of the translation invariant model to the image. This information can be interpreted as a confidence measure for the orientation estimation.

A lack of smoothness or deviation of the local structure to translation invariance leads to a decrease of the confidence measure. Curved lines occur more often in practice then straight lines. We must note that the method works on individual lines as well as on bundles of lines. We apply a local coordinate transform in such a way that the curved line becomes translation invariant, i.e. straight. The structure tensor defined on the transformed area is expressed in the original coordinates. This yields a curvature corrected confidence measure. Maximization of this confidence measure occurs for a local parabolic transformation that "closest resembles" the local structure. From the parameters of the optimal model we obtain an estimate of local curvature. This method was successfully applied in 2-D [4] and now extended to 3-D.

We apply the curvature corrected estimates to the detection of channels in a seismic volume. The occurrence of sedimentary structures are important clues for the geological model of a region. A channel is a curvilinear sedimentary structure that is characterized by its meandering morphology.

In section 2 we summarize the traditional analysis of 3D structures using the gradient structure tensor. The theory of [4] is extended to 3-D line-like structures in section 3 . Section 4 shows the results of a test of robustness of the new estimators on synthetic images and in section 5 the channel detection application is presented.

\section{The Gradient Structure Tensor}

For a local neighborhood $f(x, y, z)$ the Gradient Structure Tensor (GST) is given by eq.(1), derivative notation by indexes.

$$
\mathbf{T}_{G S}=\left[\begin{array}{lll}
\overline{f_{x}^{2}} & \overline{f_{x} f_{y}} & \overline{f_{x} f_{z}} \\
\overline{f_{x} f_{y}} & \overline{f_{y}^{2}} & \overline{f_{y} f_{z}} \\
\overline{f_{x} f_{z}} & \overline{f_{y} f_{z}} & \frac{\overline{f_{z}^{2}}}{}
\end{array}\right]
$$

Where $\overline{(.)}$ indicates some weighted local average. In [5] a closed-form analytical solution for the eigenvalues and eigenvectors of $\mathbf{T}_{G S}$ is presented. Furthermore, they show that the eigenvalues can be used define local dimensionality 
measures.

$$
C_{\text {plane }}=\frac{\lambda_{1}-\lambda_{2}}{\lambda_{1}+\lambda_{2}}, C_{\text {line }}=\frac{\lambda_{2}-\lambda_{3}}{\lambda_{2}+\lambda_{3}}
$$

These measures can differentiate between the following local structures.

$$
\begin{array}{llll}
\text { Isotrope: } & \lambda_{1} \approx \lambda_{2} \approx \lambda_{3} & C_{\text {plane }} \approx 0 & C_{\text {line }} \approx 0 \\
\text { Plane-like: } & \lambda_{1} \gg \lambda_{2} \approx \lambda_{3} & C_{\text {plane }} \approx 1 & C_{\text {line }} \approx 0 \\
\text { Line-like: } & \lambda_{1} \approx \lambda_{2} \gg \lambda_{3} & C_{\text {plane }} \approx 0 & C_{\text {line }} \approx 1
\end{array}
$$

The orientation of the eigenvectors can be used as a robust estimate of the local orientation.

\section{The GST in parabolic coordinates for 3-D line bundles}

In the previous section we stated that for line-like structures the eigenvalues of the structure tensor relate as $\lambda_{1} \approx$ $\lambda_{2} \gg \lambda_{3}$. For the simplest line-like structure, a straight line, $C_{\text {line }}=1$. However for a slightly bend line the third eigenvalue will increase, causing a decrease of the value of $C_{\text {line }}$, indicating a worser fit of the line-model. In this section we will improve this fit by incorporating curvature into the model.

We use the orthonormal set of eigenvalues of $\mathbf{T}_{G S}$ to define the local axes $u, v, w$ in such way that

$$
\lambda_{1}=\overline{f_{u}^{2}}, \lambda_{2}=\overline{f_{v}^{2}}, \lambda_{3}=\overline{f_{w}^{2}}
$$

The tangent to a line bundle is given by the third eigenvector of the GST, which we defined as the $w$-axis in eq.(3). For curved lines with a circle-symmetric cross-

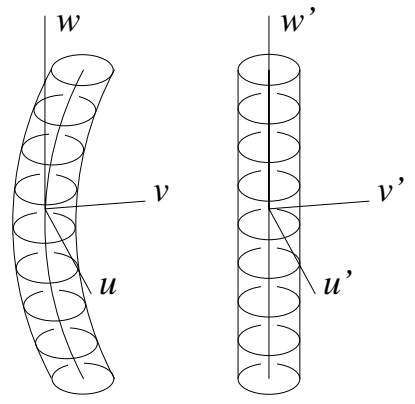

Figure 1: The transform to parabolic coordinates $u^{\prime} v^{\prime} w^{\prime}$ straightens circular bended line bundles

section (spaghetti) the 'bend-plane' is the $v w$-plane. This is not the case for less symmetrical cross-sections. Therefore the curve has two degrees of freedom $\left(\kappa_{1}, \kappa_{2}\right)$, which are the curvatures in resp. the $u w$-plane and the $v w$-plane. The total curvature $\kappa$ along the line is thus

$$
\kappa=\sqrt{\kappa_{1}^{2}+\kappa_{2}^{2}}
$$

The coordinate transform to straighten curved line bundles is given by

$$
\begin{array}{rlrl}
u^{\prime} & =u-\frac{1}{2} \kappa_{1} w^{2} & u & =u^{\prime}+\frac{1}{2} \kappa_{1}{w^{\prime}}^{2} \\
v^{\prime} & =v-\frac{1}{2} \kappa_{2} w^{2} & v & =v^{\prime}+\frac{1}{2} \kappa_{2} w^{\prime 2} \\
w^{\prime} & =w & w & =w^{\prime}
\end{array}
$$

The derivatives of $f$ expressed in the new straightened coordinates $u^{\prime}, v^{\prime}, w^{\prime}$, using the inverse coordinate transform from eq.(5), are given in eq.(6).

$$
\begin{aligned}
f_{u^{\prime}} & =u_{u^{\prime}} f_{u}+v_{u^{\prime}} f_{v}+w_{u^{\prime}} f_{w}=f_{u} \\
f_{v^{\prime}} & =u_{v^{\prime}} f_{u}+v_{v^{\prime}} f_{v}+w_{v^{\prime}} f_{w}=f_{v} \\
f_{w^{\prime}} & =u_{w^{\prime}} f_{u}+v_{w^{\prime}} f_{v}+w_{w^{\prime}} f_{w} \\
& =\kappa_{1} w f_{u}+\kappa_{2} w f_{v}+f_{w}
\end{aligned}
$$

We now consider a curved line bundle through the origin of the $u v w$-space with its rotation center in the $u v$-plane, see fig.1. Applying the traditional GST method to the $u^{\prime} v^{\prime} w^{\prime}$ space for arbitrary $\kappa_{1}, \kappa_{2}$, we get the parabolic gradient structure tensor (PGST) in eq.(7)

$$
\begin{aligned}
& \mathbf{T}_{P G S}=\left[\begin{array}{ccc}
\overline{f_{u^{\prime}}^{2}} & \overline{f_{u^{\prime}} f_{v^{\prime}}} & \overline{f_{u^{\prime}} f_{w^{\prime}}} \\
\overline{f_{u^{\prime}} f_{v^{\prime}}} & \overline{f_{v^{\prime}}^{2}} & \overline{f_{v^{\prime} f_{w^{\prime}}}} \\
\overline{f_{u^{\prime}} f_{w^{\prime}}} & \overline{f_{v^{\prime}} f_{w^{\prime}}} & \overline{f_{w^{\prime}}^{2}}
\end{array}\right]= \\
& {\left[\begin{array}{ccc}
\overline{f_{u}^{2}} & 0 & 0 \\
0 & \overline{f_{v}^{2}} & 0 \\
0 & 0 & Q+\overline{f_{w}^{2}}-K
\end{array}\right]}
\end{aligned}
$$

with the abbreviations

$$
\begin{aligned}
Q \equiv & \left(\kappa_{1}-\kappa_{10}\right)^{2} a+\left(\kappa_{1}-\kappa_{10}\right)\left(\kappa_{2}-\kappa_{20}\right) b \\
& +\left(\kappa_{2}-\kappa_{20}\right)^{2} c \\
\kappa_{10} \equiv & \frac{b e-2 c d}{4 a c-b^{2}} \\
\kappa_{20} \equiv & \frac{b d-2 a e}{4 a c-b^{2}} \\
K \equiv & a \kappa_{10}^{2}+b \kappa_{10} \kappa_{20}+c \kappa_{20}^{2} \\
a \equiv & \overline{w^{2} f_{u}^{2}}, b \equiv 2 \overline{w^{2} f_{u} f_{v}}, c \equiv \overline{w^{2} f_{v}^{2}} \\
d \equiv & 2 \overline{w f_{u} f_{w}}, e \equiv 2 \overline{w f_{v} f_{w}}
\end{aligned}
$$

Due to the symmetry $f(u, v, w)=f(u, v,-w)$, we get

$$
\begin{aligned}
& \overline{f_{u^{\prime}} f_{w^{\prime}}}=\kappa_{1} \overline{w f_{u}^{2}}+\kappa_{2} \overline{w f_{u} f_{v}}+\overline{f_{u} f_{w}}=0 \\
& \overline{f_{v^{\prime}} f_{w^{\prime}}}=\kappa_{1} \overline{w f_{u} f_{v}}+\kappa_{2} \overline{w f_{v}^{2}}+\overline{f_{v} f_{w}}=0 \\
& \overline{f_{u^{\prime}} f_{v^{\prime}}}=\overline{f_{u} f_{v}}=0
\end{aligned}
$$

The last equation requires mirror symmetry with respect to some axis in the $u v$-plane. The eigenvalues of $\mathbf{T}_{P G S}$ are

$$
\lambda_{1}=\overline{f_{u}^{2}}, \lambda_{2}=\overline{f_{v}^{2}}, \lambda_{3}=Q+\overline{f_{w}^{2}}-K .
$$


We see that the third eigenvalue can be minimized by choosing $\kappa_{1}=\kappa_{10}$ and $\kappa_{2}=\kappa_{20}$, causing $C_{\text {line }}$ to maximized. Therefore we interpret $\kappa_{10}$ and $\kappa_{20}$ as the actual curvatures. In the limit $\kappa_{1} \rightarrow 0, \kappa_{2} \rightarrow 0$, we return to the traditional analysis where $\lambda_{3}=\overline{f_{w}^{2}}$. Substituting $\kappa_{1}=\kappa_{10}, \kappa_{2}=\kappa_{20}$, the tensor gets the "curvature corrected" eigenvalues

$$
\lambda_{1}=\overline{f_{u}^{2}}, \lambda_{2}=\overline{f_{v}^{2}}, \lambda_{3}=\overline{f_{w}^{2}}-K
$$

\section{Test results}

To measure the robustness of the new estimators for confidence and curvature, we created an 3-D image of a torus and added different levels of Gaussian noise, see figure 5. The torus has a Gaussian profile, which makes the object band-limited. We choose the width of the torus $\sigma=2.0$ voxel. Since the grey values of the test object are bounded, $0 \leq f(x, y, z) \leq 1$, we use the following definition for the signal-to-noise ratio

$$
\begin{aligned}
S N R & =20 \log _{10}\left(\frac{\max (f)-\min (f)}{\sigma_{n}}\right) d B \\
& =-20 \log _{10}\left(\sigma_{n}\right) d B
\end{aligned}
$$

where $\sigma_{n}$ is the standard deviation of the noise.

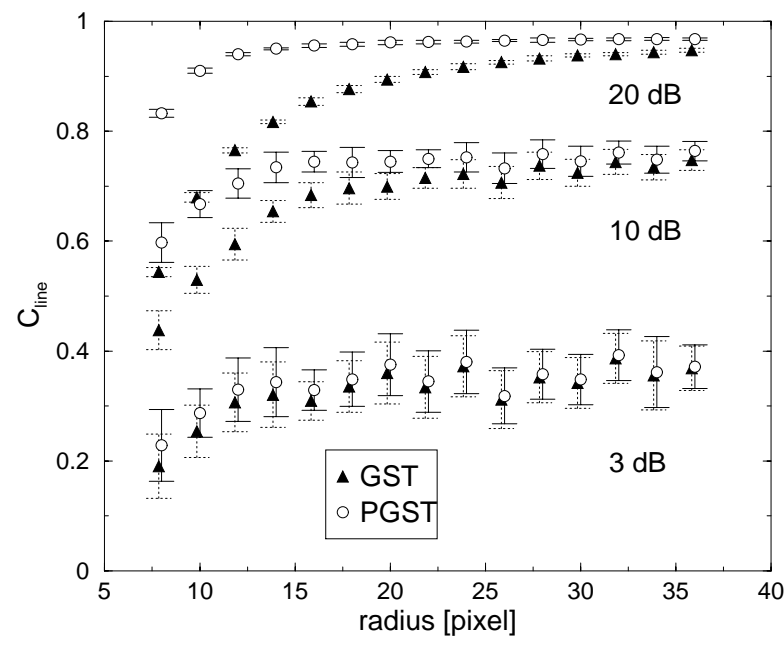

Figure 2: A comparison of the traditional (GST) and the curvature corrected (PGST) confidence estimation. The average confidence estimation is depicted as a function of the radius of the torus at different different SNR. The error-bars indicate the standard deviation.

The results of the confidence and curvature estimation on the torus image are depicted in resp. figure 2 and 3 . The average and the standard deviation for each point in these

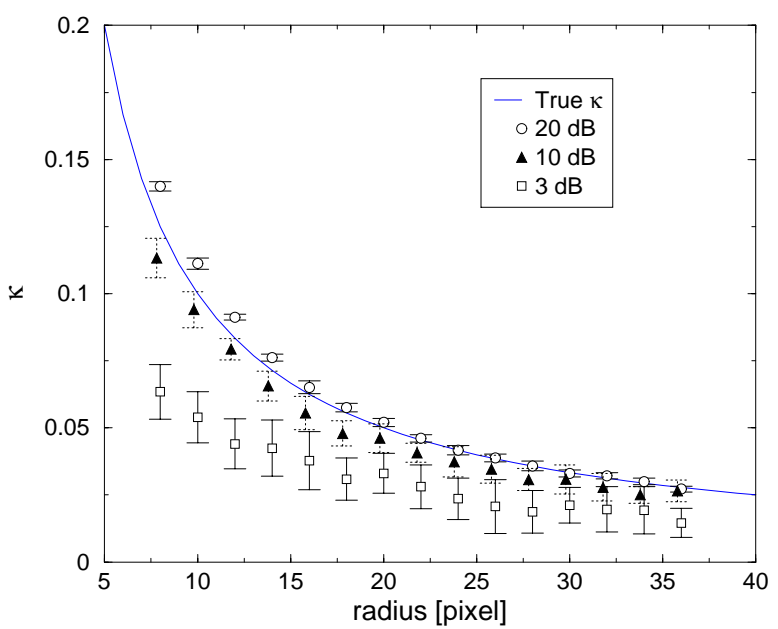

Figure 3: Average curvature estimation on the torus-image for different SNR. The error-bars indicate the standard deviation.

figures is taken over 12 measurements. For both the gradient regularisation and for the local averaging of the structure tensor a Gaussian window was used. We applied a gradient smoothing $\sigma_{g}=1.0$ and a tensor smoothing $\sigma_{T}=4.0$ for all measurements. The scale of the tensor smoothing is chosen such that an optimal SNR for the measurement is achieved. More details about the selection of the scale for the tensor smoothing are given in the discussion at the end of the paper.

In figure 2 we see that at 10 and $20 \mathrm{~dB}$, the curvature corrected method yields a significant improvement in the confidence estimation. At $3 \mathrm{~dB}$, however, there is no significant improvement, which means that there is not enough signal energy for a confident fit of the model. As a consequence, the curvature estimation at $3 \mathrm{~dB}$ is not reliable. The curvature estimation at $10 \mathrm{~dB}$ has an average relative error of $12 \%$.

Note that $C_{\text {line,gst }} \leq C_{\text {line,pgst }}$ for all radii. This can be explained by the fact that adding an extra parameter to the model, always results in a better fit. The increasing of the bias in the curvature estimation for decreasing SNR, is explained in appendix A.

\section{Application}

For the automatic detection of channels in 3-D migrated seismic reflection data, we model channels as curvilinear structures meandering in 3-D space. The seismic volume containing a channel that we are going to analyze is visualized by 2-D cross-sections in figure 6 and 7 . The xy-axes are spatial and the z-axis spans the amplitude of the reflec- 
tion of an acoustic wavelet as a function of the travel time from the surface. The z-axis can be inverted to depth if the velocity of the acoustic wave is known at each depth.

The meandering nature of channels suggests that the curvature corrected model gives a more accurate description of a channel than the traditional straight model. Therefore we expect that the curvature corrected confidence estimation yields a significantly higher value than the traditional confidence estimation.

As a preprocessing step, we create an attribute volume by computing the standard deviation within a window of $\left(w_{x}=w_{y}=5, w_{z}=9\right)$ voxels, for each voxel in the seismic volume. A cross-section of this volume, corresponding to figure 7 (a), is depicted in figure 10 . We performed the confidence estimation on the attribute volume, with both the GST and the PGST method. We applied a gradient smoothing $\sigma_{g}=1.0$ and a tensor smoothing $\sigma_{T}=3.5$ for all measurements.

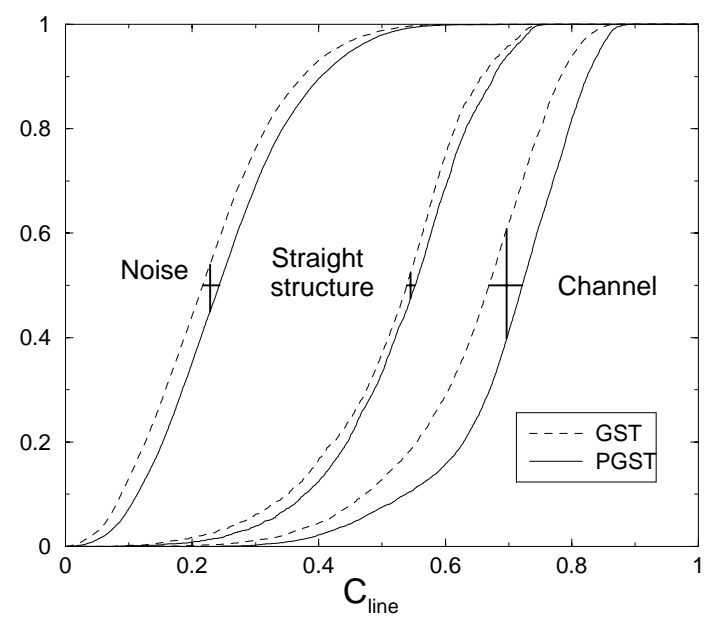

Figure 4: Cumulative distribution of the confidence estimation of selected parts of the seismic volume.

To analyze the result, we defined three regions in the seismic volume. One that contains all data points that have manually labeled 'channel'. The second one consist of points from region with no seismic events, and represents the noise in the data. The last regions contains a straight sedimentary structure. For all three regions we created the cumulative distribution of the confidence estimate, which are displayed in figure 4 .

\section{Discussion and conclusions}

Inspection of figure 4 leads to several conclusions. As already found in the test results the PGST confidence estimate always yields a higher value than the GST confidence esti- mate. We convenience denote the confidence improvement as

$$
\Delta C=C_{\text {line,pgst }}-C_{\text {line,gst }} .
$$

The significance of $\Delta C$ along the channel depends on the noise.

We assume that the noise has the same distribution for position in the volume. The improvement $\Delta C_{n}=0.03$ in the noise region gives an estimation of the improvement due to noise $(S N R=-\infty)$. However, the value of $\Delta C_{n}$ is not constant for all $C_{\text {line }}$ values. Consider a straight line with $S N R=\infty$, then $\Delta C=0$ thus $\Delta C_{n}=0$. In the "straight structure" region, the curvature correction improves the fit by giving a better description of the noise. Therefore we have $\Delta C_{n}=0.01$ at $C_{\text {line }}=0.55$. We can now conclude that the noise contribution to $\Delta C=0.05$ in the channel region $\Delta C_{n}<0.01$, and the improvement is therefore due to a better description of the meandering structure of the channel.

Segmentation of channels requires information from larger scales, because on On the scale we used for the tensor smoothing there is often not enough information to do a confident channel segmentation. Therefore it is necessary to include larger scale information. Increasing the local estimation-window size isotropically decreases the SNR. The signal of a linear structure in a 3-D isotropic window increase linearly with the radius $R$ of the window. The noise increases with $R^{3 / 2}$, assuming uncorrelated normally distributed white noise. The solution is to increased the scale only along the channel. This can be done by steering an adaptive window using the estimated orientation and curvature.

\section{Acknowledgments}

This research is partly supported by Senter, Agency of the Ministry of Economic Affairs of the Netherlands, the Royal Netherlands Academy of Arts and Sciences (KNAW), and the Rolling Grants program of the Foundation for Fundamental Research in Matter (FOM).

\section{References}

[1] M. Kass, A. Witkin. Analyzing oriented patterns, Computer Vision Graphics and Image Processing, 1987.37:p. 362-385.

[2] Hans Knutsson. Representing Local Structure Using Tensors, The 6th Scandinavian Conference on Image Analysis, Oulu, Finland, pp. 244-251, June 19-22, 1989. 
[3] L. Haglund. Adaptive multi-dimensional filtering, Ph.D Thesis, Linkoping University, Linkoping, 1992.

[4] P.W. Verbeek, L.J. van Vliet, J. van de Weijer. Improved Curvature and Anisotropy Estimation for Curved Line Bundles, Proceedings of ICPR'98, Brisbane, Australia, 17-20 August 1998

[5] G.M.P. van Kempen, N. van de Brink, L.J. van Vliet, P.W. Verbeek, M. van Ginkel, H. Blonk. The application of a Local Dimensionality Estimator to the analysis of 3-D microscopic network structures, The 11th Scandinavian Conference on Image Analysis, Kangerlussuaq, Greenland, pp. 447-455, June 7-11, 1999

\section{Appendix A}

From figure 3 it is clear that noise introduces a bias in the curvature $\kappa$ estimation; the estimated curvature is smaller than the actual curvature. This can be explained by examining $\kappa_{10}, \kappa_{20}$.

$$
\begin{gathered}
\kappa_{10}=\frac{\overline{w^{2} f_{u} f_{v}} \overline{w f_{v} f_{w}}-\overline{w^{2} f_{v}^{2}} \overline{w f_{u} f_{w}}}{\overline{w^{2} f_{u}^{2}} \overline{w^{2} f_{v}^{2}}-\left(\overline{w^{2} f_{u} f_{v}}\right)^{2}} \\
\kappa_{20}=\frac{\overline{w^{2} f_{u} f_{v}} \overline{\frac{w f_{u} f_{w}}{w^{2} f_{u}^{2}}} \overline{\overline{w^{2} f_{v}^{2}}}-\overline{\left(\overline{w^{2} f_{u} f_{v}}\right)^{2}} \overline{\frac{f_{v} f_{w}}{2}}}{\kappa=\sqrt{\kappa_{10}^{2}+\kappa_{20}^{2}}}
\end{gathered}
$$

The terms $\overline{w^{2} f_{i}^{2}}, i=u, v$ increase when noise is added, while the other terms do not change. Since the increasing terms appear quadratic in the denominator and linear in the nominator, the curvature estimation becomes smaller when noise is added. The sign of $\kappa_{10}, \kappa_{20}$ is lost in eq.14.

\section{Appendix B}

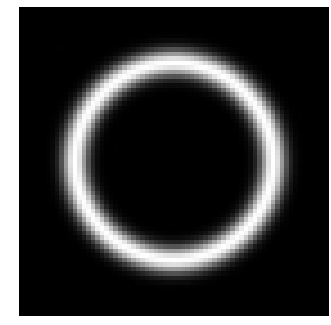

(a)

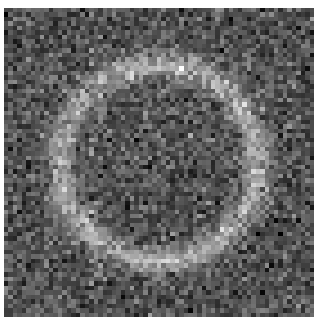

(c)

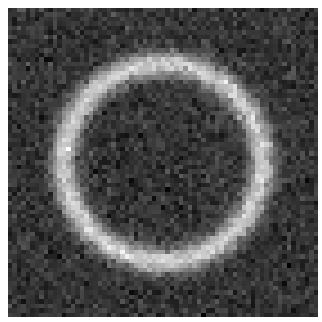

(b)

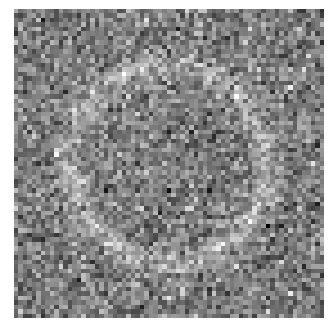

(d)
Figure 5: A slice through the center of the torus-image (a), with Gaussian noise added to SNR $20 \mathrm{~dB}$ (b), $10 \mathrm{~dB}$ (c), 3 $\mathrm{dB}(\mathrm{d})$.

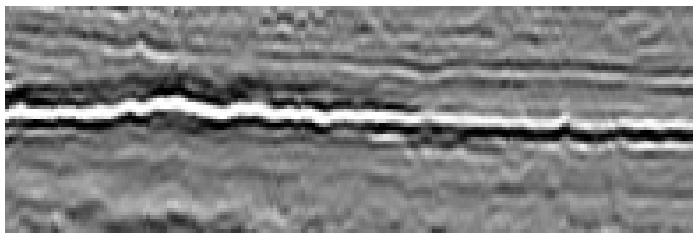

Figure 6: A cross-section of the seismic volume: t-axis plotted along the center-line of the channel. 


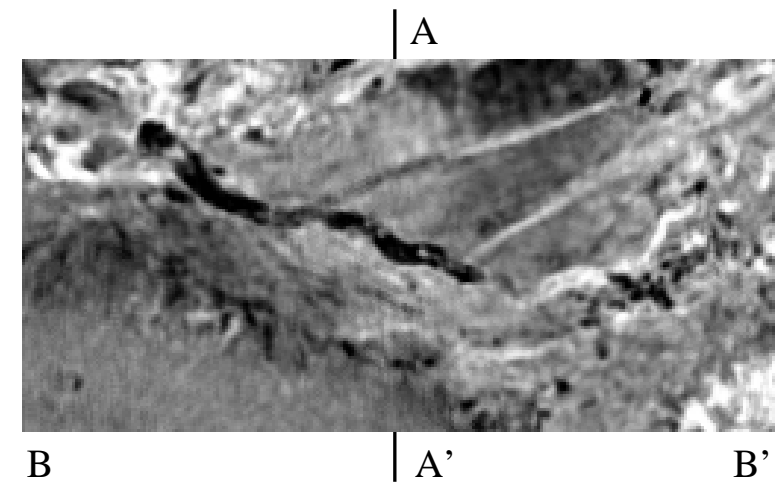

(a)

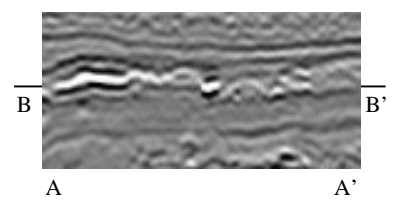

(b)

Figure 7: Slices of the seismic volume: (a) xy-slice, (b) ytslice, centered in the $\mathrm{x}$-direction.

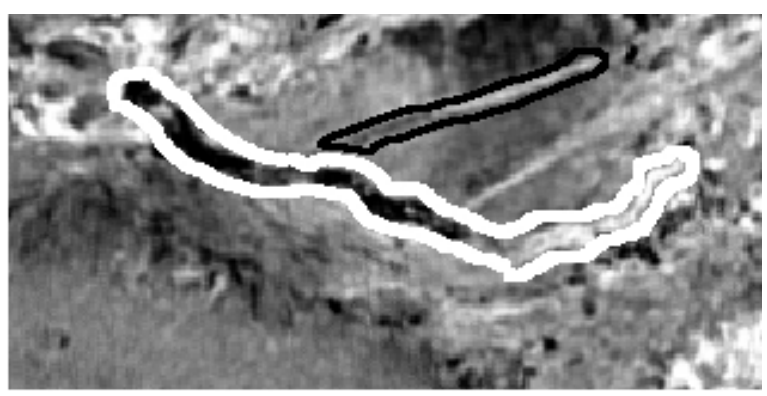

Figure 8: The same as figure 7 (a) with the channel region outlined in white and the straight sedimental structure outlined in black.

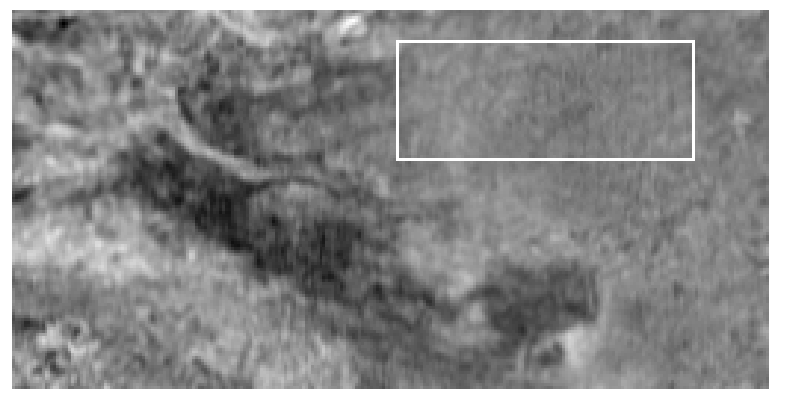

Figure 9: A xy-slice of the seismic volume with the noise region outlined in white.

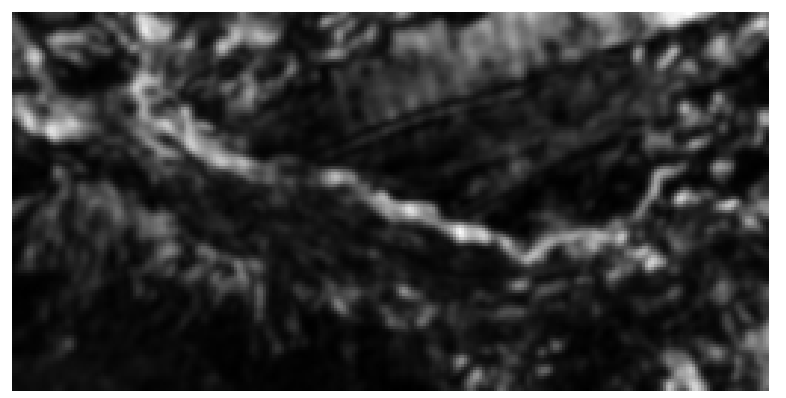

Figure 10: xy-slice through the 3-D attribute volume, corresponding the figure 7 (a). 